Provided for non-commercial research and education use. Not for reproduction, distribution or commercial use.

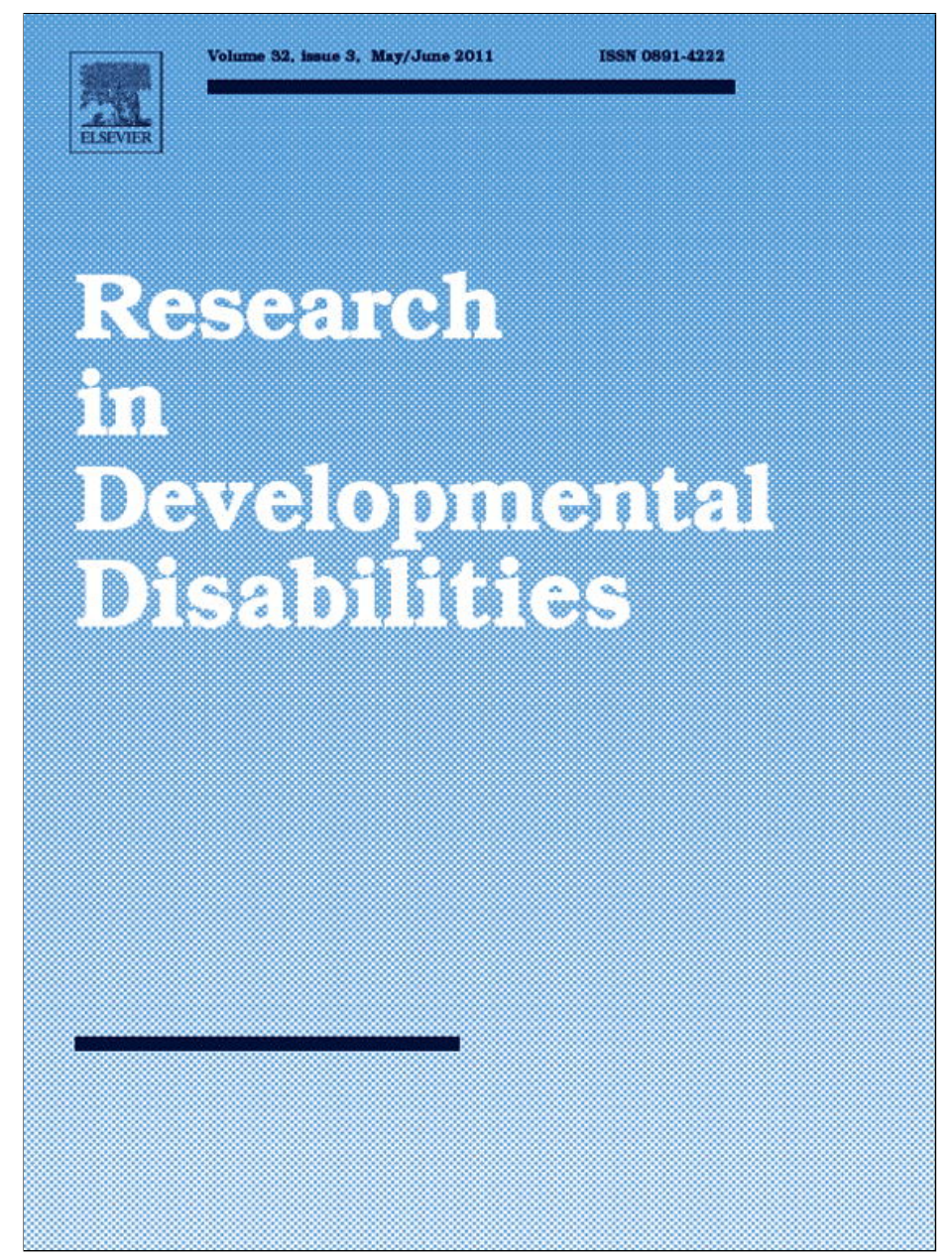

This article appeared in a journal published by Elsevier. The attached copy is furnished to the author for internal non-commercial research and education use, including for instruction at the authors institution and sharing with colleagues.

Other uses, including reproduction and distribution, or selling or licensing copies, or posting to personal, institutional or third party websites are prohibited.

In most cases authors are permitted to post their version of the article (e.g. in Word or Tex form) to their personal website or institutional repository. Authors requiring further information regarding Elsevier's archiving and manuscript policies are encouraged to visit:

http://www.elsevier.com/copyright 


\title{
Williams syndrome hypersociability: A neuropsychological study of the amygdala and prefrontal cortex hypotheses
}

\author{
Liliana Capitão $^{\mathrm{a}}$, Adriana Sampaio ${ }^{\mathrm{a}, *}$, Montse Férnandez ${ }^{\mathrm{a}, \mathrm{b}, \mathrm{c}}$, Nuno Sousa $^{\mathrm{c}}$, \\ Ana Pinheiro ${ }^{a}$, Óscar F. Gonçalves ${ }^{a}$ \\ ${ }^{a}$ Neuropsychophysiology Lab, CIPsi, School of Psychology, University of Minho, 4710-057 Braga, Portugal \\ ${ }^{\mathrm{b}}$ Genetic Molecular Unit, Galician Public Foundation of Genomic Medicine, University of Santiago de Compostela, Spain \\ ${ }^{\mathrm{c}}$ Life and Health Sciences Research Institute, University of Minho, Braga, Portugal
}

\section{A R T I C L E I N F O}

\section{Article history:}

Received 21 December 2010

Accepted 8 January 2011

Available online 12 February 2011

\section{Keywords:}

Williams syndrome

Neurodevelopment

Hypersociability

\begin{abstract}
A B S T R A C T
Individuals with Williams syndrome display indiscriminate approach towards strangers. Neuroimaging studies conducted so far have linked this social profile to structural and/or functional abnormalities in WS amygdala and prefrontal cortex. In this study, the neuropsychological hypotheses of amygdala and prefrontal cortex involvement in WS hypersociability was explored using three behavioral tasks - facial emotional recognition task, a social approach task and a go no/go task. Thus, a group 15 individuals with Williams syndrome was compared to two groups of normal developing individuals - a group of 15 individuals matched for chronological age (CA) and 15 individuals matched for mental age (MA), and sex. Individuals with WS present a specific impairment in recognizing negative facial expressions and do not display impairments in response inhibition when compared with typically developing groups. Although these findings partially support the amygdala contribution to WS hypersociability, we found that general cognitive functioning predicted this performance. Additionally, individuals with WS did not differ from both CA and MA groups in the recognition of angry facial expressions, a finding suggesting that they are actually able to identify stimuli associated with social threat. Overall, the results seem to indicate that this social profile must be understood within a developmental framework.
\end{abstract}

(c) 2011 Elsevier Ltd. All rights reserved.

\section{Introduction}

Williams syndrome (henceforth WS) is a rare neurodevelopmental disorder caused by a hemideletion on chromosome 7q11.23 (Peoples et al., 2000). One of the most striking features of individuals with WS is their distinct social-affective profile, characterized by high sociability, disinhibition, over-friendliness (Bellugi, Adolphs, Cassady, \& Chiles, 1999; Klein-Tasman \& Mervis, 2003) and strong empathy (Klein-Tasman \& Mervis, 2003).

The precise etiology of WS hypersociability is still unknown; however, neuroanatomical, genetic/molecular and behavioral/ neuropsychological studies have been providing important clues regarding the neural and genetic mechanisms underlying WS social phenotype. Of the different brain areas, the amygdala and prefrontal cortex have emerged more consistently as the two main neuroanatomical regions that can be hypothesized to be associated with WS hypersocial phenotype.

\footnotetext{
* Corresponding author. Tel.: +351253 604613.

E-mail address: adriana.sampaio@psi.uminho.pt (A. Sampaio).
} 
Amygdala, a component of the neural network underlying social cognition, plays an important role in processing emotional and social-related stimuli (Adolphs, 1999) and is related to monitoring environmental events such as danger (Amaral, 2002). These findings have led some researchers to propose that an abnormal amygdala processing may be responsible for the hypersocial behavior typical of WS phenotype (e.g., Galaburda \& Bellugi, 2000; Reiss et al., 2004). However, structural magnetic resonance neuroimaging has produced inconsistent results regarding volume or gray matter density of amygdala in WS (Chiang et al., 2007; Jernigan, Bellugi, Sowell, Doherty, \& Hesselink, 1993; Martens, Wilson, Dudgeon, \& Reutens, 2009; Reiss et al., 2004). Functional studies found that people with Williams syndrome showed greater amygdala activation in response to threatening scenes than to threatening faces (Meyer-Lindenberg et al., 2005). Taking into account that amygdala plays a key role in monitoring danger; these authors proposed that its reduced activation in individuals with WS in response to threatening faces might contribute to their reduced fear of strangers and consequent social disinhibition. Moreover, recent fMRI studies also found an abnormal activation of the amygdala during face processing (Haas et al., 2009; Paul et al., 2009), providing additional evidence indicating that failure to recruit this area during face processing may underlie WS high sociability. Indeed, this hypothesis of an amygdala involvement in WS hypersociability is also congruent with data showing that individuals with lesions in this area are abnormally friendly towards others (Adolphs, 1999), suggesting impairment in evaluating the potential threat of objects or organisms in the environment prior to approaching them. Also, these individuals fail to recognize emotions such as fear, anger, disgust and sadness (Adolphs, 1999) and present a positive bias when evaluating trustworthiness and approachability of faces in natural poses (Adolphs, Tranel, \& Damasio, 1998).

Together with abnormal patterns of amygdala activation during face processing, evidence of an abnormal connection between the amygdala and the prefrontal cortex (in particular the orbitofrontal cortex), suggests also a prefrontal involvement in the WS social phenotype (Meyer-Lindenberg et al., 2005). Thus, an abnormal reactivity of prefrontal areas has been implicated in WS social knowledge. For instance, in the study of Meyer-Lindenberg and colleagues (2005), WS individuals (as opposed to controls) showed a task-invariant pattern: orbitofrontal (OFC) cortex was not differentially activated, and both medial prefrontal cortex (MPFC) and dorsolateral prefrontal cortex (DLPFC) were equally responsive during face and scene matching. However, the demanding nature of those tasks was not similar (face matching task was more difficult than the non-social scene matching task). To overcome this limitation, Muñoz et al. (2009) used the same group of participants but using a more cognitively exigent task (Muñoz et al., 2009). Again, results were consistent with the previous findings; specifically, they evidenced increased amygdala reactivity to non-social relevant aversive stimuli in individuals with WS, regardless of cognitive load; and no prefrontal activation differences between conditions (contrasting with a greater prefrontal reactivity specifically in left DLPFC, left OFC and MPFC in the control group as a function of task difficulty). The results provide further evidence of disruption in amygdala-prefrontal circuitry in individuals with WS (Muñoz et al., 2009). Additionally, Mobbs et al. (2007) proposed that an abnormal frontostriatal circuit, in conjunction with abnormal connectivity between the amygdala and OFC, might be associated with the hypersocial profile characteristic of individuals with WS. The hypothesis of a prefrontal impairment being associated with the hypersociability found in WS has also been proposed by a set of behavioral studies (Porter, Coltheart, \& Langdon, 2007; Rhodes, Riby, Park, Fraser, \& Campbell, 2009) and is also congruent with specific structural brain patterns found in WS in terms of volumetric difference in the frontal lobes specifically, volumetric changes (Ewart et al., 1993; Gothelf et al., 2008; Meyer-Lindenberg et al., 2004; Reiss et al., 2004) and abnormal patterns of gyrification (Gaser et al., 2006; Schmitt et al., 2002).

Taken together, these characteristics suggest that abnormalities in amygdala and prefrontal cortex regulation may contribute to WS hypersocial profile. Whereas amygdala dysfunction is possibly related to an incapacity to recognize threat in the environment and thus to properly determine the trustworthiness and approachability of unfamiliar individuals (consistent with a positive bias in evaluating trustworthiness and approachability of faces in natural and emotional poses (Jones et al., 2000; Martens et al., 2009)), proposal of prefrontal cortex abnormalities is based upon clinical similarity with patients with prefrontal lesions (e.g., disinhibition in several circumstances, including social contacts, and displaying rigid and inflexible behaviors) (Semel \& Rosner, 2003) and the fact that, despite the nonrewarding nature of their social relationships (e.g., problems in making and sustaining relationships and are often rejected by their peers), individuals with WS are continuously attracted to social stimuli, thus revealing some insensitivity to punishing and negative experiences.

Thus, the objective of the present study is to test amygdala and prefrontal cortex involvement in WS hypersociability with a neuropsychological approach, using three different behavioral tasks: an emotional recognition task; a social approach task; a judgment and response inhibition task. The two following hypotheses were tested:

(a) The amygdala hypothesis - if people with WS display manifestations of amygdala dysfunction similar to those with acquired damage to this structure, they should display impairment in recognizing negative emotions (specifically, anger, fearful and sadness). Also, they should judge photos of unfamiliar individuals as highly approachable, not rating happy expressions as more approachable than negative ones (especially those displaying in anger).

(b) The prefrontal hypothesis - if the abnormal social approach in WS is consistent with patterns of acquired frontal lobe impairment, individuals with WS should display impaired response inhibition on go/no-go tasks (Liddle, Kiehl, \& Smith, 2001). 
Table 1

Socio-demographic characteristics of the groups.

\begin{tabular}{|c|c|c|c|c|c|c|c|c|}
\hline & \multicolumn{3}{|l|}{ WS $(N=15)$} & & \multicolumn{2}{|l|}{$\mathrm{CA}(N=15)$} & \multicolumn{2}{|l|}{$\operatorname{MA}(N=15)$} \\
\hline & $\mathrm{M}(\mathrm{SD})$ & & Range & & $\mathrm{M}(\mathrm{SD})$ & Range & $\mathrm{M}(\mathrm{SD})$ & Range \\
\hline Age & $15(3.8)$ & & $9-21$ & & $14.87(3.9)$ & $8-21$ & $7.47(2.5)$ & 4-14 \\
\hline \multirow[t]{3}{*}{ Full Scale IQ } & $52.6(10.4)$ & & $40-74$ & & $108.87(9.2)$ & $92-123$ & $107.73(13.8)$ & $87-130$ \\
\hline & & \multicolumn{3}{|c|}{ WS $(N=15)$} & & $\mathrm{CA}(N=15)$ & \multicolumn{2}{|c|}{$\mathrm{MA}(N=15)$} \\
\hline & & Mdn & & Range & Mdn & Range & Mdn & Range \\
\hline Level of education & & 8 & & $2-9$ & 1 & $0-8$ & 1 & $(0-8)$ \\
\hline Sócio-economical status & & 3 & & $1-4$ & 3 & $2-4$ & 3 & $2-4$ \\
\hline \multicolumn{9}{|l|}{ Sex } \\
\hline Male & & 8 & & $53.33 \%$ & 8 & $53.33 \%$ & 8 & $53.33 \%$ \\
\hline Female & & 7 & & $46.67 \%$ & 7 & $46.67 \%$ & 7 & $46.67 \%$ \\
\hline
\end{tabular}

\section{Method}

\subsection{Participants}

Study participants included 15 individuals with Williams syndrome ( 8 males and 7 females) ( $m e a n \pm S D$ age, $15 \pm 3.761$; age-range: 9-21 years), 15 typically developing individuals matched for chronological age (CA) and sex (mean \pm SD age, $14.87 \pm 3.94$; age-range: $8-21$ years) and15 typically developing individuals matched for mental age (MA) and sex (mean \pm SD age, $7.47 \pm 2.50$; age-range: $4-14$ years). The mean Full Scale IQ was 52.6 ( \pm 10.398$)$ for SW, 109.13 ( \pm 9.141$)$ for CA group and $107.73( \pm 13.755)$ for MA group. Persons with WS were recruited at the Genetic Medical Institute (Portugal) and the Genomic Foundation in Galicia (Spain). WS diagnoses were made by FISH confirmation of elastin gene deletion (Ewart et al., 1993). Controls were typically developing individuals without evidence of psychiatric, neurological disorder or cognitive impairment. After a complete description of the study, each participant (or their parents) gave written informed consent regarding their participation in the study via consent forms.

Table 1 displays socio-demographical characteristics of the sample. There are no group differences with respect to socioeconomical status - Graffar index $\left[\chi^{2}=(2)=.519 ; p=.775\right]$, although differing in education level $\left[\chi^{2}(2)=19.614 ; p<.001\right]$, and in IQ $\left[\chi^{2}(2)=19.734 ; p<.001\right]$. Finally, WS and CA groups did not differ in chronological age $[t(2)=19.714 ; p=.914]$.

\subsection{Neurocognitive assessment}

To assess general cognitive functioning, participants with 4 and 5 years were administered the Wechsler Preschool and Primary Scale of Intelligence - Revised (WPPSI-R; Wechsler \& Corporation, 2006), participants between 8 and 16 years of age were administered the Wechsler Intelligence Scale for Children - Third Edition (WISC-III; Wechsler, 1991) and subjects over 16 years old were administered the Wechsler Adult Intelligence Scale - Third Edition (WAIS-III; Wechsler, 1997). However, it is important to note that children from the mental age control group between 6 and 7 years were only administered four subtests from the WISC-III: Block design, Information, Figure completion and Similarities. The Full Scale IQ was computed using the following formula: 1.6 (sum of the standardized tests) + 36 [for children with 6 years] and 1.6 (sum of the standardized tests) + 32 [for children with 7 years] (Kaufman, Kaufman, Balgopal, \& McLean, 1996). Mental age of individuals with WS was inferred individually according to Full Scale IQ (Terman, 1916).

\subsection{Materials and stimuli}

The procedure, materials and stimuli were presented for each task (emotion recognition task, approachability task and go/no-go tasks). Tasks were administered in this exact order and were performed in Portuguese and Spanish versions, according to the participants nationality. All tasks were compiled and ran using Superlab 4.0.7.

\subsubsection{Facial expression recognition task}

2.3.1.1. Stimuli. The facial expression recognition task was designed to evaluate emotion recognition abilities in relation to different stimuli with positive, negative and neutral valences. This task consisted in the presentation of faces of 12 individuals ( 6 adults, 3 teenagers and 3 children), displaying five different and mutually exclusive emotions - anger, fear, neutral, happiness and sadness.

The adult pictures were taken from the Karolinska Directed Emotional Faces (Lundqvist et al., 1998) whereas the photographs from children and teenagers were purposely developed with children from two Portuguese schools. None of the participants had beards, mustaches, earrings, braces or eyeglasses. The photographs were normalized for size and luminance (when necessary) and the background details of all photographs were masked out in white using the Adobe Photoshop CS3; so, distracting cues such as hair and clothes were eliminated, enhancing the focus on the facial expression (see Fig. 1). 

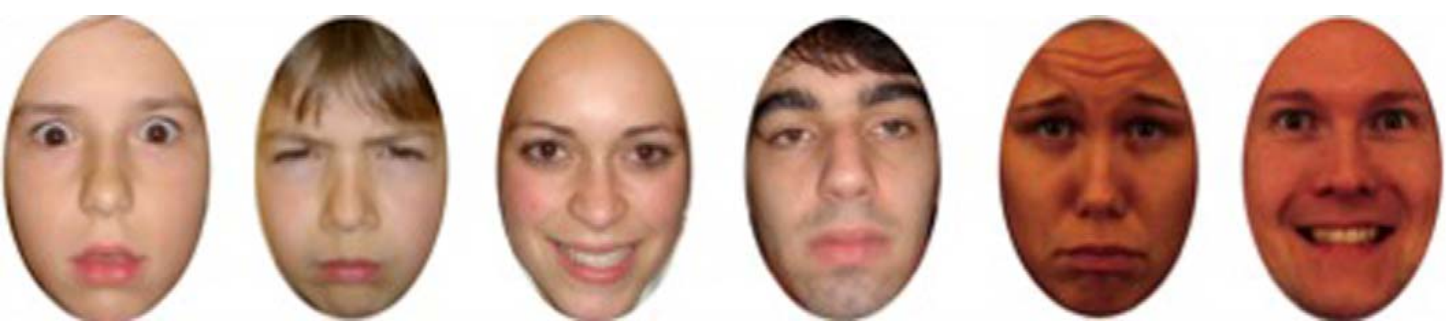

Fig. 1. Examples of photos used in the emotion recognition and approachability tasks.

All stimuli were validated with a population of 25 children from a primary school (mean \pm SD age, $9.16 \pm 0.37$; age-range: 9-10 years) to establish that a typically developing group correctly labeled emotions. The criterion used to validate the stimuli was the one suggested by Ekman (Ekman, Friesen, \& Press, 1976): 90\% of the photographs had to be correctly identified by at least $80 \%$ of the raters.

2.3.1.2. Procedure. Prior to testing, to acclimatize participants with the task, they were asked to label the examiner's facial expressions and to produce on their own face the five facial expressions: anger, neutral, fear, happiness and sadness. Then, 10 practice trials were performed to familiarize the participants with the task. These trials consisted of the presentation of faces of two adult individuals depicting the five expressions (one man and one woman) that were not used elsewhere in the experiment. After the practice trial, participants were presented with one block containing a total of 60 trials. Participants were asked to label which emotion was displayed by the depicted individual. The experimenter read the five options that appeared on the computer screen - happy, neutral, scared/fearful, angry and sad (corresponding to the five emotions depicted in the photographs) and then asked the participants to choose one of them. The photographs remained on screen until the experimenter keyed the participant's answer. There were two possibilities for the fear emotion - scared and fearful, since these emotions are almost interchangeable and some children could interpret some expressions as scared and others as fearful. Thus, in order to avoid this kind of ambiguity, both terms were accepted. Also, the term neutral expression was replaced (when necessary) by "normal expression" (because is not conceptualized by the children as a "typical" emotion). The order of trials was randomized for each participant.

\subsubsection{Approachability task}

2.3.2.1. Stimuli. This task was intended to evaluate approachability ratings in relation to facial stimuli displaying different emotions (happy, neutral, scared/fearful, angry and sad). The approach scale was similar to previously ones (Frigerio et al., 2006). The photographs used in this task were the same used in the facial expression recognition task; including 60 trials of 12 individuals depicting five facial expressions. The fact that the background details of the photographs were masked out was particularly important in this task. Indeed, the effect of confounding variables such as different hairdos, shape of ears, was controlled to avoid prejudiced judgments, based on factors other than the face depicting the emotion.

2.3.2.2. Procedure. Prior to testing, 10 practice trials were performed to familiarize the participants with the task. These trials consisted of faces of two adult individuals depicting five expressions (one male and one female) that were used in the practice trials of the first task. Participants were asked whether they would like to interact (talk and/or play) with the pictured individuals. If the participants answered positively they were then asked to what extent they wished to interact: "a lot" (score of 4) or "a little" (score of 3). If they replied negatively they were further asked to quantify their answer as "probably not" (score of 2) or "definitely not" (score of 1 ). Participants were told that there was no right or wrong answer, to avoid a social desirability bias.

\subsubsection{Go/no-go tasks}

The go-no tasks used in this study were adapted from the standard go/no-go paradigm that has been used for decades to test behavioral inhibition (Costantini \& Hoving, 1973). These tasks consisted of three emotional tasks and one non-emotional task. The emotional go/no-go tasks were adapted from similar ones used in studies with typically developing populations (Reynolds \& Jeeves, 1978; Schulz et al., 2007). The go/no go tasks had the same structure, timing parameters and response demands and provided identical measures of behavioral inhibition but to different types of stimuli: emotional and nonemotional. Additionally, the use of affective stimuli enabled analysis of performance in response to cues of different emotional valences (e.g., happy, fearful and angry). Thus, these tasks not only provide a measure of behavioral inhibition, but also of the emotional modulation of this inhibition (Drevets \& Raichle, 1998).

Both tasks required participants to press as rapidly as possible a key (M) whenever they see the target stimuli (go cues), while withholding responses to non-target stimuli (no-go cues). Both the emotional and the non-emotional go/no-go tasks consisted of 100 stimuli, of which 90 (90\%) were go cues and 10 (10\%) were no-go cues. The high frequency of go cues creates thus a tendency to respond that is required to be inhibited for no-go cues, thus providing a measure of the ability to inhibit responses. In each trial a single face appeared and remained on the center of the screen until the participant keyed an answer or after $3 \mathrm{~s}$. Trial order was randomized for each individual. 
2.3.3.1. Stimuli. Three different emotional go/no-go tasks were presented. These tasks differed only in the no-go cues; one of the tasks had happy faces as the no-go stimuli; other had scared faces and still other had angry ones. Happy expressions were selected because they are thought to induce approachable behaviors, whereas fearful and angry expressions signal the presence of threat (Adolphs, Russell, \& Tranel, 1999), thus inducing avoidant behaviors. It is important to note that fearful faces alert to some threat other than the expresser, hence signalling danger in an indirect way.

The go-cues for all these tasks were neutral faces from adults, children and teenage models (the same 90 neutral photographs were presented in all tasks - 30 men, 30 women, 8 teenage girls, 7 teenage boys, 7 young girls and 8 young boys). The happy non-go cues were from 4 women, 3 men, 1 teenage girl, 1 young girl and 1 young boy. The angry faces were from 4 women, 1 teenage boy, 1 teenage girl and 1 young girl. Finally, the scared photographs were from 3 women, 4 men, 1 teenage boy, 1 teenage girl and 1 young boy.

The adult photographs were selected from the Karolinska Directed Emotional Faces, whereas the children and teenage photographs were taken specifically for this study, along with the faces for the first task. The faces depicting happiness, fear and anger were all validated (in the two validations studies mentioned above), while some neutral ones were not (the only neutral faces validated were those also used in the first task). Indeed, as it is easy to distinguish neutral faces from those displaying positive and negative emotions, and since the number of neutral faces was elevated, we assumed the validity of the stimuli. The photographs were normalized for size and luminance (when necessary) and were masked out in white, using the Adobe Photoshop CS3.

The letters $\mathrm{A}$ and $\mathrm{X}$ of the same size and colour (black) served as the stimuli for non-emotional go and no-go cues, respectively, and this task consisted in the presentation of 90 letters " $A$ " and 10 letters "X".

2.3.3.2. Procedure. Prior to testing, a practice task was performed to familiarize the participants with this task. This practice test consisted in the presentation of 20 faces ( 15 neutral and 5 happy). The tasks were performed in the following sequence: go/no-go task with scared faces, go/no-go task with happy faces, go/no-go task with letters and go/no-go task with angry faces. Participants were instructed to press as rapidly as they could a key of the keyboard (in this case the "M") when they see the go cues, while withholding responses for the non-go cues. These instructions were better explained or simplified when necessary to guarantee that the participants understood it.

\subsection{Data analysis}

Data were computed using the SPSS software for Windows 16.0 version. A mixed analysis of variance was used to determine differences in total correct scores (for task 1) and approach ratings (for task 3 ) between WS group and individuals matched for chronological (CA) and mental age (MA). Thus, group (three levels: WS subjects, CA and MA groups) was used as the between-subject factor and Emotion/Approach ratings (five levels: happy, neutral, sad, angry and scared) as the within factor. Also, a mixed analysis of covariance was conducted to analyze the effect of Full Scale IQ (FSIQ) in the emotion recognition task. The criteria of normality (Kolmogorov-Smirnov and Shapiro-Wilk tests), variance homogeneity (Levene and Box tests), sphericity (Mauchly's test) and homogeneity for regression slopes (for analysis of covariance) were evaluated. When sphericity was violated, Greenhouse Geisser corrections were reported. If an interaction effect between Group and Emotion was found, then a one-way analysis of variance was conducted to test the mean difference between groups. A linear regression was further conducted to analyze the effect of FSIQ on total correct scores. The criteria for sample (15 subjects for predictor), independence (Durbin Watson), multicolinearity and singularity (Colinearity Statistics), outliers (Cook's Distance), linearity, independence and homoscedasticity of residuals were evaluated.

Additionally, for the go/no-go task with letters, a one-way analysis of variance was conducted to determine differences in correct responses, false positives, omissions and time reactions. Also, an analysis of covariance was conducted to analyze the effect of Full Scale IQ (FSIQ) in this task. A linear regression was further conducted to see if the FSIQ predicted performance in these dependent variables.

A mixed analysis of variance was conducted to analyze performance (i.e., correct responses, false positives, omissions and time reactions) in the emotional go/no-go tasks. Group (three levels: WS subjects, CA and MA controls) was used as the between-subject factor and Emotion (three levels: happy, angry and scared) as the within factor. If an interaction effect between Group and Emotion was found, then a one-way analysis of variance was conducted to test the mean difference between groups. Further, a linear regression was conducted to explore a possible influence of FSIQ in performance of emotional go/no-go tasks taken together. A spearman rank correlation was also used to correlate performance obtained in the go/go-no go task with letters and in emotional go/no-go tasks. A $p$ value less than .05 was assumed to denote a significant difference.

\section{Results}

\subsection{Emotion recognition task}

Total correct scores (that is, the mean proportion of correct responses) were analyzed using a mixed analysis of variance with Group as a between-groups factor (three levels: WS, CA and MA) and Emotion as a within-subjects factor (five levels: happy, neutral, sad, angry and scared). 

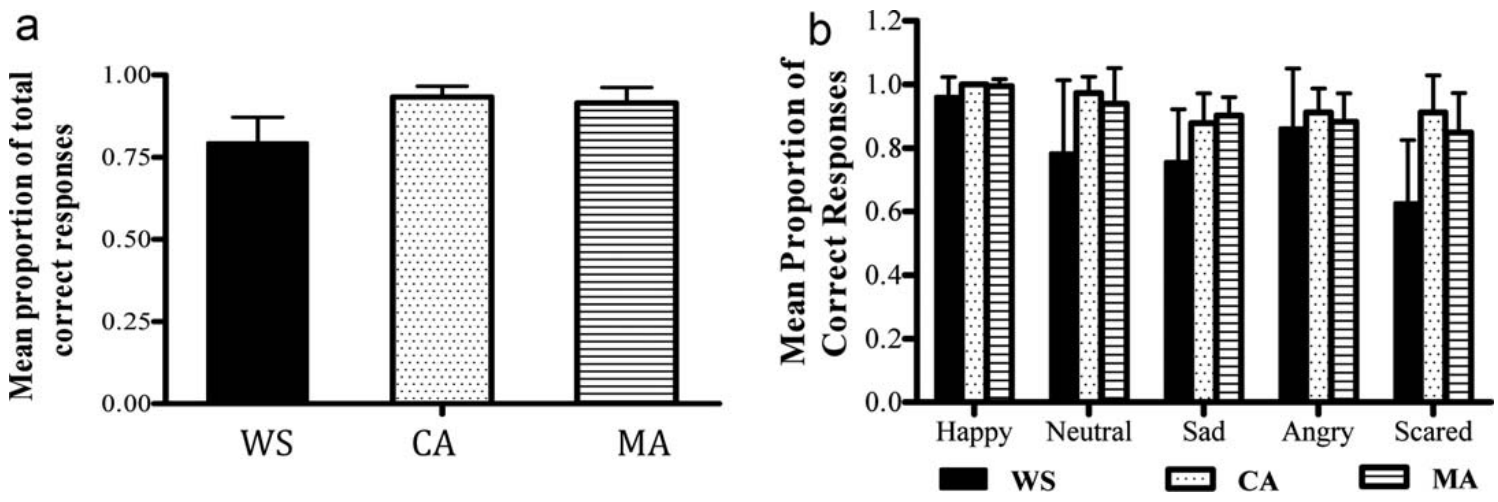

Fig. 2. (A) Mean proportion of total correct responses on the emotion recognition task and (B) mean proportion of correct responses on the emotion recognition task for each expression.

The analysis revealed a significant main effect for Group $[F(2,42)=28.090, p<.001]$, Emotion $[F(3.078,129.281)=14.352$, $p<.001]$ and a significant Group by Emotion interaction $[F(6.156,129.281)=3.071, p<.01]$. The main effect for group occurred because the CA and MA groups outperformed SW group ( $p<.001$ for both comparisons), with respect to overall emotion recognition abilities (that is, the mean proportion of correct responses given to all facial expressions taken together).

Follow-up analyses were conducted with one-way analysis of variance, to explore the significant Group by Emotion interaction. When evaluating performance in relation to each facial expression, there was a group effect for all emotions but for angry faces, in which no differences between groups was observed $[F(2,44)=.583, p=.563]$. Significant group differences were evident in relation to happy faces $[F(2,44)=4.569, p<.05]$, with WS group performing worse than chronological age-matched controls ( $p<.05$, .96 vs. .1, respectively), but at the same level as MA group $(p=.067)$; additionally, no differences were found between the CA and MA groups $(p=.929)$. When considering sad faces, group differences were observed $[F(2,44)=6.930$, $p<.01$ ], with the WS group performing worse than the CA group $(p<.05, .76$ vs. .88, respectively) and controls matched for mental age ( $p<.01, .76$ vs. .90, respectively); again, no differences were found between the CA and MA groups $(p=.850)$. In relation to scared faces, there was a group effect $[F(2,44)=14.797, p<.001]$, with WS group performing worse than

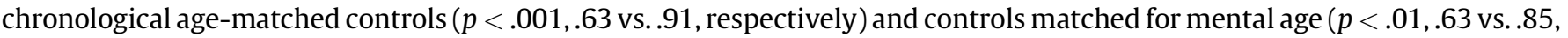
respectively); again, no differences were found between the CA and MA groups $(p=.528)$. Finally, in relation to neutral faces, there were significant differences between groups $[F(2,44)=6.757, p<.01]$, with WS group performing worse than chronological age-matched controls ( $p<.01, .78$ vs. .97, respectively) and controls matched for mental age ( $p<.01, .78$ vs. 94 , respectively) and no differences between typically developing groups were found $(p=.832)$ (see Fig. 2).

Since the participants of this study differ in terms of FSIQ (with subjects with WS having mild to moderate cognitive impairments), a one-way analysis of covariance was performed to control the effect of this variable on total correct scores. The covariate(i.e.,FSIQ)wasnotsignificantly related tothisdependentvariable $[F(1,41)=.147 ; p=.704]$. Additionally, aftercontrolling for FSIQ a group effect for total correct scores remained $(F(2,41)=3.296 ; p<.05)$. This finding is in accordance with the fact that subjects with WS had a worse performance that individuals matched for mental age when considering total correct scores.

When a mixed analysis of covariance was computed with Group as a between-groups factor (three levels: WS, CA and MA), Emotion as a within-subjects factor (five levels: happy, neutral, sad, angry and scared) and FSIQ as a covariate, no main effects emerged. Specifically, main effects for Emotion $[F(3.071,125.898)=.801, p=.498]$, Group by Emotion interaction $[F(3.071$, $125.808)=.357, p=.788]$ andFSIQbyEmotioninteraction $[F(6.141,128.898)=.551, p=.773]$ werenotobserved. Finally, wedidnot find a main effect for FSIQ alone $[F(1,41)=.023, p=.880]$, although it was found a main effect for group $[F(2,41)=4.028, p<.05]$.

To further explore the relationship between FSIQ and performance in the emotion recognition task, a linear regression was conducted with FSIQ as predictor variable and total correct scores as dependent variable. This model was significant $[F(1$, $43)=43.060, p<.001]$, explaining $50 \%\left(R_{\text {adj }}^{2}=48.9 \%\right)$ of variance. Accordingly, FSIQ significantly predicted correct total scores $(t=6.562, p<.001)$, being positively related to this measure $(\beta=.707)$.

\subsection{Error misclassifications}

Table 2 shows the percentages of expressions incorrectly labeled as happy, neutral, sad, angry or scared for each group when performing the emotion recognition task. Error classifications indicated that happy faces were the stimuli most easily

Table 2

Mean percentage (and standard deviation) of misclassifications for each of the five emotions on the emotion recognition task.

\begin{tabular}{llllll}
\hline & Happy & Fear & Neutral & Sad & Angry \\
\hline SW & $0.05(0.11)$ & $0.11(0.09)$ & $0.32(0.19)$ & $0.18(0.16)$ & $0.25(0.21)$ \\
CA & $0.01(0.03)$ & $0.06(0.06)$ & $0.12(0.14)$ & $0.06(0.06)$ & $0.06(0.06)$ \\
MA & $0.05(0.06)$ & $0.06(0.06)$ & $0.10(0.12)$ & $0.18(0.16)$ & $0.11(0.12)$ \\
\hline
\end{tabular}



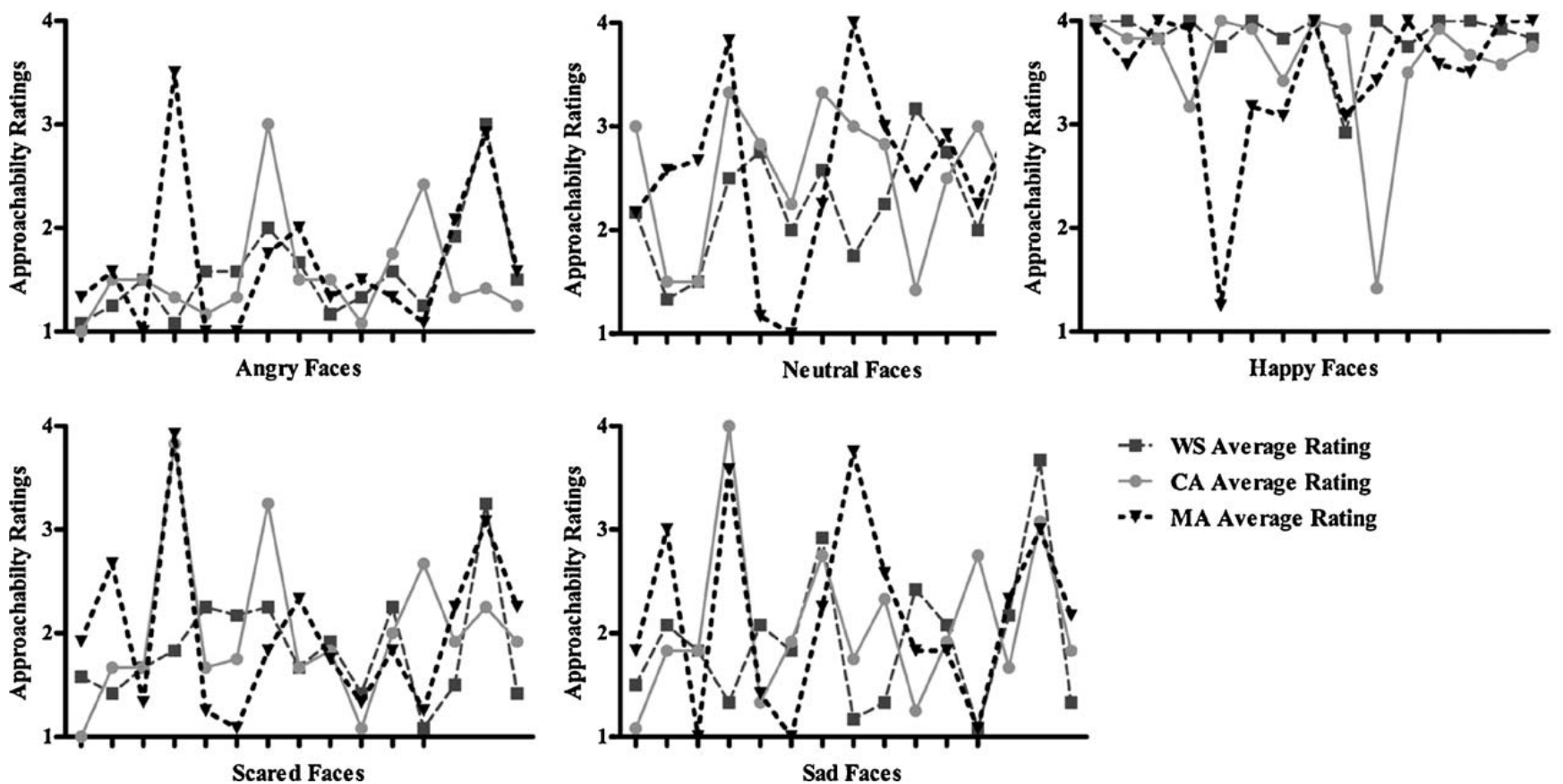

Fig. 3. Mean approachability ratings for each facial stimulus of the five expressions $(12 \times 5)$. WS = subjects with Williams syndrome; $\mathrm{CA}=$ chronological agematched controls; MA = mental age-matched controls.

identified in all groups and that individuals with WS had the highest percentage of misclassifications (with exception for happy and sad faces, in which displayed a similar profile to the MA group). Also, this group commonly misclassified expressions as neutral and angry.

\subsection{Social approach task}

Fig. 3 shows mean approach ratings for each group on the approach task. Approachability ratings are shown separately for each emotion (happy, neutral, sad, angry and scared/fearful). Emotion recognition affected performance on the social approach task, specially for the WS group, with mean approach ratings being more positive for happy expressions and less positive for negative ones (angry, scared and sad). Also, this group rated neutral faces as medium approachable with the other typically developing groups showing more individual variation in their responses, although it is visible that they rated the happy faces as more approachable than the remaining ones.

Mean approachability ratings were analyzed using a mixed analysis of variance with Group as a between-groups factor (three levels: WS, CA and MA) and Emotion as a within-subjects factor (five levels: happy, neutral, sad, angry and scared). It was found a significant main effect for Emotion $[F(2.710,113.805)=129.5055 ; p<.001]$. However, no Group effect $[F(2$, $42)=.161, p=.851]$ nor a Group by Emotion interaction $[F(5.419,113.805)=1.493 ; p=.163]$ were observed.

The social approach results were further re-analyzed using data derived uniquely from those facial expressions, which were correctly identified in the emotion recognition task, to see if the approachability ratings were influenced by the ability to correctly identify facial expressions. The results obtained were similar, indicating that approachability ratings were not influenced by the ability to correctly identify facial expressions. Again, a main effect for Emotion [F(2.732, $114.743)=116.751 ; p<.001]$ was obtained and no Group $[F(2,42)=.149, p=.862]$ nor for Group by Emotion $[F(5.464$, $114.743)=1.377 ; p=.234]$ effects were observed.

\subsection{Go/no-go tasks}

Correct responses (i.e., correct go and no-go trials), false positives (i.e., commission errors), omissions (i.e., not responding to go stimuli) and reaction times (on go trials) on the go/no-go task with letters ${ }^{1}$ were analyzed with a one-way analysis of variance. In this task, groups differed with respect to correct responses $[F(2,43)=6.653, p<.01]$ and omissions $[F(2$, $43)=6.653, p<.05]$, but did not differ regarding the number of false positives $[F(2,43)=2.133, p=.131]$. The Scheffe post hoc test revealed that the CA group outperformed WS group when considering both correct responses (1 vs. .99, respectively, $p<.01$ ) and omissions ( 0 vs. .13, respectively, $p<.1$ ), but performed at the same level as the MA group ( $p=.491$ for both measures). Also, WS and MA groups had an equivalent performance when considering both correct responses and omissions $(p=.066$ and $p=.491$, respectively) (see Fig. 4).

\footnotetext{
${ }^{1}$ Only 14 individuals with WS were considered for this analysis.
} 

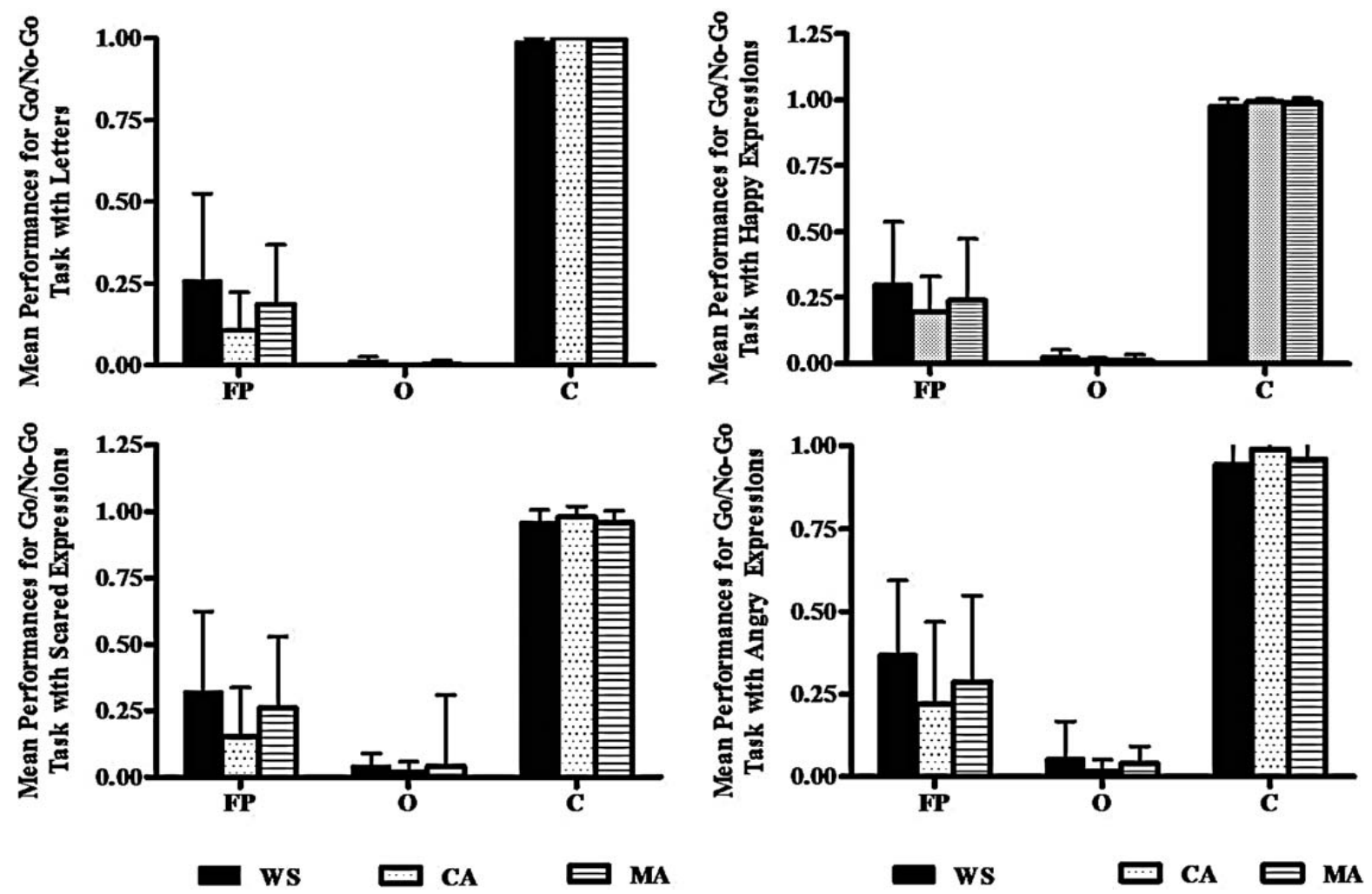

Fig. 4. Mean false positives (FP), omissions (O) and correct responses (C) for non-emotional (with letters) and emotional go/no-go tasks (with happy, scared and angry faces).

To evaluate if FSIQ affected results in this task, an analysis of covariance was performed to control the effect of this variable in the number of omissions and correct scores. The covariate was not related to the number of omissions $[F(1$, $40)=.270, p=.606]$ and, after controlling for FSIQ, no differences between the three groups were observed $[F(2,40)=1.015$, $p=.371]$. Similarly, results indicated that the FSIQ was not significantly related to total correct $s c o r e s[F(1,40)=.270$, $p=.606]$ and, after controlling the effect of this variable, no group effect for corrected scores was found $[F(2,40)=1.015$, $p=.371]$.

A linear regression analysis was further conducted to explore these relationships, with FSIQ being the predictor variable and correct responses and omissions the dependent ones. The first model (including correct scores as the dependent variable) was significant $[F(1,42)=11.299, p<.01]$, explaining $21.2 \%\left(R_{\mathrm{adj}}^{2}=19.3 \%\right)$ of variance. Accordingly, the FSIQ significantly predicted correct responses in this task $(t=3.361, p<.01)$, being positively related to this measure $(\beta=.460)$.

The second model (with omissions as the dependent variable) was also significant $[F(1,42)=11.299, p<.01]$, explaining $21.2 \%\left(R_{\mathrm{adj}}^{2}=19.3 \%\right)$ of variance. Accordingly, FSIQ significantly predicted omissions $(t=-3.361, p<.01)$, being, as expected, negatively related to this measure $(\beta=-.460)$.

When analyzing mean reaction times for the go/no-go task with letters, significant differences between groups were found $[F(2,41)=4.163, p<.05]$. But, surprisingly, the WS group performed equally to both CA $(p=.159)$ and MA group $(p=.729)$. Differences were found between CA and MA groups: individuals with MA were slower when performing this task $(p<.05)$. This finding is especially relevant, since performance of individuals with WS was not affected by their fine motor coordination difficulties (Gagliardi, Martelli, Burt, \& Borgatti, 2007).

Correct responses, false positives, omissions and time reactions in emotional go/no-go tasks ${ }^{2}$ were analyzed using a mixed analysis of variance, with Group as a between-groups factor (three levels: WS, CA and MA) and Emotion as a withinsubjects factor (three levels: happy, angry and scared).

In relation to correct responses, no main effects were observed for Group $[F(1.310,52.410)=4.077 ; p=.024]$, Emotion $[F(1.310,52.410)=1.192, p=.294]$, or interaction between Emotion and Group $[F(2.620,52.410)=1.200, p=.316]$ or between Emotion and FSIQ $[F(1.310,52.410)=1.199, p=.294]$. Accordingly, after controlling for FSIQ, no group effect was found $[F(1.310,52.410)=1.199, p=.294]$ as well as a FSIQ effect, when considered alone $[F(1,40)=1.511, p=.226]$.

When analyzing false positives, we did not observed effects of Emotion $[F(1.580,66.346)=1.028, p=.348]$, Group $[F(2$, $42)=.028, p=.972]$ and Emotion by Group interaction $[F(3.159,66.346)=1.186, p=.323]$. In relation to omissions, we no effect for Emotion $[F(1.007,42.285)=1.323, p=.257]$, Group $[F(2,42)=1.306, p=.282]$ and Emotion by Group interaction $[F(2.014,42.285)=1.092, p=.345]$ were found.

\footnotetext{
${ }^{2}$ In the emotional go/no-go task with angry faces, only 14 participants with WS were considered.
} 
When analyzing reaction times for the emotional go/no-tasks, a main effect for Emotion $[F(1.532,62.811)=5.086, p<.05]$ was evident, but no significant Group $[F(1,41)=.897, p=.416]$ or Group by Emotion interaction $[F(3.064,62.811)=.539$, $p=.661]$ was observed.

Finally, there was a significant correlation between false positive of emotional tasks taken together and those verified in the go/no-go task with letters ( $\left.\mathrm{rs}_{\mathrm{p}}=.554, p<.001\right)$. Correct responses and omissions from both this type of tasks were also significantly correlated $\left(\mathrm{rs}_{\mathrm{p}}=.427, p<.01\right.$ and $\mathrm{rs}_{\mathrm{p}}=.407, \mathrm{p}<.01$, respectively).

\section{Discussion}

The aim of this study was to explore emotion recognition abilities, social approach judgments and response inhibition in individuals with WS, in order to provide a neuropsychological approach concerning the amygdala and prefrontal cortex neuropsychological hypotheses underlying WS social profile.

In relation to the emotion recognition task, we found that individuals with WS performed worse than chronological and mental age matched typically individuals when recognizing neutral, scared and sad faces. These data are partially in accordance with previous studies assessing emotion recognition abilities in individuals with WS (Gagliardi et al., 2003; Lacroix, Guidetti, Rogé, \& Reilly, 2009; Porter et al., 2007; Skwerer et al., 2009; Tager-Flusberg \& Sullivan, 2000), namely showing that individuals with WS perform worse than chronological age-matched controls in emotion recognition tasks (Gagliardi et al., 2007; Porter et al., 2007). However, regarding happy facial expressions, we observed that individuals with WS performed at the same level as individuals matched for mental age. These differences in results may be possibly related to the utilization of different measures to compute mental age. Indeed, Lacroix et al. (2009), using verbal mental age to match a group of individuals with WS and with autism, obtained results similar to those obtained here, namely that individuals with WS had a worse performance than individuals with autism and controls matched for mental age when recognizing fear and sadness.

In the approachability task, the performance of the WS group did not differ from both control groups, thus evidencing a normal rank order of approach. These findings are in stark contrast with data obtained by Jones et al. (2000) and more recently by Martens et al. (2009) of abnormal positive ratings in the WS group towards trustworthy and untrustworthy faces. However, it is important to note that the stimuli used in these studies did not include different facial expressions (instead, individuals displayed natural expressions), which may account for these disparities. Additionally, the results obtained in this study are inconsistent with those reported by Frigerio et al. (2006), namely, individuals with WS rated happy faces as significantly more approachable than chronological- and mental age-matched controls and the negative faces as significantly less approachable than both control groups. Indeed, we observed that even when individuals with WS are not able to correctly identify facial expressions, they seem to be able to determine the level of approachability of strangers, which is in agreement with the evidence of similar ratings in the approach task in WS and individuals matched for chronological age when considering the emotions correctly identified (Porter et al., 2007).

Regarding the amygdala dysfunction hypothesis, although these results may suggest that individuals with WS present a specific impairment in recognizing negative facial expressions, which, in turn, may contribute to their strong urge to make social interactions, and thus corroborating the amygdala hypothesis (e.g., Meyer-Lindenberg et al., 2005; Muñoz et al., 2009; Paul et al., 2009), the results of the present study bring additional information that do not fit within this framework and deserves further discussion. First, we observed a positive association between FSIQ and performance in the emotion recognition task, suggesting that intellectual functioning accounts for this ability. Second, individuals with WS did not differed from both CA and MA groups when recognizing angry facial expressions, indicating that they are actually able to identify the emotion that more directly signal social threat. Finally, amygdala dysfunction hypothesis does not extend to approachability ratings presented by individuals with WS, since they do not differ from both typically developing groups. A possible hypothesis is that individuals with this syndrome are able to distinguish approachable from non-approachable persons, despite not being able to act in accordance with this social knowledge.

Therefore, in the response inhibition task (go-no go tasks) we observed that individuals with WS did not show impairment in response inhibition, since comparison of the number of false positives (the primary measure of behavioral inhibition) for both emotional and non-emotional tasks was similar in the groups under analysis. Also, despite having a worse performance than individuals matched for chronological age when considering correct responses and omissions in the go/no-go task with letters, no differences between WS and MA group were found. Further, these disparities disappeared when the effect of FSIQ was controlled, thus demonstrating that differences in performance could not be directly attributable to impairment in prefrontal cortex. Still, no differences between groups were found for correct responses and omissions in emotional go/no-go tasks. The moderate correlation between false positives of emotional and non-emotional tasks supports the idea that these tasks provide an identical measure of response inhibition, although towards different cues. So, one could speculate that individuals with WS have preserved response inhibition abilities, towards both type of stimuli.

Results obtained with the non-emotional go/no-go task contrast with those obtained by Mobbs et al. (2007). These authors showed a preserved performance of individuals with WS in a go/no-go task with letters in terms of accuracy, but slower mean time reactions in comparison with typically developing subjects. It is important to note that false positives were not directly evaluated in this study, thus limiting comparisons with findings obtained here. Indeed, the only report assessing social disinhibition in WS and Down syndrome, was performed by Porter et al. (2007). Despite the task used by these authors was similar to the go/no-go tasks used here, the stimuli were shapes with happy and sad faces. Performance of individuals 
with WS was below the level expected on the basis of their level of intellectual functioning; however, no direct comparisons with typically developing subjects were carried out. Still, this finding was interpreted as evidence suggesting that the hypersociability of individuals with WS could be attributable to impairment in the frontal lobe. However, as the authors themselves acknowledged, a direct link between frontal lobe dysfunction and the abnormal social profile verified in WS could not be inferred based on this type of task. Therefore, the present study tried to create a task that would directly evaluate the relationship between prefrontal (executive) functioning - in particular response inhibition, and indiscriminate real-world social approach in WS. To achieve this, real-life stimuli of unfamiliar persons were included in all no/no-go tasks. However, these stimuli and corresponding setting may have not approximated the real-life situations where hypersocial behaviors of WS manifest. Indeed, in contrast to emotion recognition and approachability tasks - which intended to evaluate awareness, a construct insensible to the setting where it is evaluated, the go/no-go tasks of this study aimed to evaluate behavior, a more complex construct that is inherently dependent of contextual circumstances. Furthermore, executive deficits are a characteristic of WS (Menghini, Addona, Costanzo, \& Vicari, 2010) and are more prone to be manifested in nonstructured environments (Powell \& Malia, 2003).

Even though we observed that individuals with WS do not show impairment in response inhibition for both emotional and non-emotional tasks, these results should not be considered robust enough to exclude the possibility of a prefrontal involvement in WS social phenotype. Moreover, this evidence highlights the difficulty in creating a task that would evaluate behavior in a rigorous way and simultaneously unravel the anatomical areas known to be responsible of social inhibition, namely OFC, a region critical for withholding responses in simple go/no-go discrimination tasks (Schoenbaum, Nugent, Saddoris, \& Setlow, 2002).

\section{Conclusions}

In sum, the current study has shown that individuals with WS present a specific impairment in recognizing negative facial expressions. These findings although consistent with amygdala dysfunction were no longer observed when controlled for the effect cognitive functioning, suggesting that this ability is strongly related to intellectual abilities. Moreover, we found that individuals with WS did not differ from both CA and MA groups when recognizing angry facial expressions, an important finding evidencing that individuals with this syndrome are actually able to identify the emotion more directly signalling social threat. Regarding the prefrontal contribution to WS hypersocial phenotype, we observed that individuals with WS do not show impairment in response inhibition for both emotional and non-emotional tasks and thus is inconsistent with this hypothesis.

The present results do not fit entirely with amygdala and prefrontal hypothesis and most of all, emphasize that rather than considering WS hypersociability according to within a lesion model, we should consider this profile taking into account a neurodevelopmental framework and thus, future studies should be carried out in order to assess longitudinal trajectories of social and emotional development in WS. Indeed, our results indicate that modular brain structures (prefrontal and amygdala) are not exclusively responsible for fundamental aspects of social behavior in early developmental disorders (Bauman, Lavenex, Mason, Capitanio, \& Amaral, 2004).

Subsequent studies in this domain should also consider the relationship between cognitive/social representations and behavior in individuals with WS, since they do not seem to make use of their knowledge about social interactions. Indeed, despite having perceptions of approachability that not differ from those displayed by typically developing subjects (as evidenced in the present study), these individuals do not seem to act accordingly to this awareness. An explanation for this phenomenon can be interpreted in light of the "somatic marker hypothesis" of Damasio (1994). This hypothesis posits that when humans experience a specific emotion, the brain records this state in cell patterns from neural and hormonal feedback, and this information is subsequently used to guide behavior appropriately (e.g., indicating whether to approach or withdraw from a situation based on predictions about rewarding/punishing consequences). So, one hypothesis could be that individuals with WS do not experience the bodily sensations (i.e., somatic markers) associated with negative emotions, which causes inappropriate behaviors in social interactions (Damasio, 1999). Also, this hypothesis may account for their apparent insensitiveness in relation to punishing experiences, since they continue be attracted by social stimuli despite experience difficulties in this realm. These predictions are actually in accordance with recent findings showing that individuals with WS are less electrodermally responsive and show more heart deceleration than controls when presented with facial stimuli (Doherty-Sneddon, Riby, Calderwood, \& Ainsworth, 2009; Skwerer et al., 2009). Further studies, employing physiological measures (e.g., skin conductance analysis) during emotion recognition/approachability/go/no-go tasks, should be conducted to test this somatic marker hypothesis in individuals with WS.

\section{Acknowledgment}

This research was supported by the grants PIC/IC/83290/2007 from Fundação para a Ciência e Tecnologia (Portugal).

\section{References}

Adolphs, R. (1999). Social cognition and the human brain. Trends in Cognitive Sciences, 3, 469-479.

Adolphs, R., Russell, J., \& Tranel, D. (1999). A role for the human amygdala in recognizing emotional arousal from unpleasant stimuli. Psychological Science, 10, 167. 
Adolphs, R., Tranel, D., \& Damasio, A. (1998). The human amygdala in social judgment. Nature, 393(6684), $470-474$.

Amaral, D. (2002). The primate amygdala and the neurobiology of social behavior: Implications for understanding social anxiety. Biological Psychiatry, $51,11-17$. Bauman, M., Lavenex, P., Mason, W., Capitanio, J., \& Amaral, D. (2004). The development of social behavior following neonatal amygdala lesions in rhesus monkeys. Journal of Cognitive Neuroscience, 16(8), 1388-1411.

Bellugi, U., Adolphs, R., Cassady, C., \& Chiles, M. (1999). Towards the neural basis for hypersociability in a genetic syndrome. Neuroreport, 10 (8), 1653.

Chiang, M., Reiss, A., Lee, A., Bellugi, U., Galaburda, A., Korenberg, J., et al. (2007). 3D pattern of brain abnormalities in Williams syndrome visualized using tensorbased morphometry. Neuroimage, 36, 1096-1109.

Costantini, A., \& Hoving, K. (1973). The effectiveness of reward and punishment contingencies on response inhibition. Journal of Experimental Child Psychology, 16, 484-494.

Damasio, A. R. (1994). Descartes' error: emotion, reason, and the human brain. New York: GP Putman.

Damasio, A. R. (1999). The Feeling of What Happens: Body, Emotion and the Making of Consciousness. London: Heinemann.

Doherty-Sneddon, G., Riby, D., Calderwood, L., \& Ainsworth, L. (2009). Stuck on you: Face-to-face arousal and gaze aversion in Williams syndrome. Cognitive Neuropsychiatry, 1, 1-14.

Drevets, W., \& Raichle, M. (1998). Reciprocal suppression of regional cerebral blood flow during emotional versus higher cognitive processes: Implications for interactions between emotion and cognition. Cognition and Emotion, 12, 353-385.

Ekman, P., Friesen, W., \& Press, C. (1976). Pictures of facial affect (Vol. 21, pp. ). ). Palo Alto, CA: Consulting Psychologists Press.

Ewart, A. K., Morris, C. A., Atkinson, D., Jin, W., Sternes, K., Spallone, P., et al. (1993). Hemizygosity at the elastin locus in a developmental disorder, Williams syndrome. Nature Genetics, 5, 11-16.

Frigerio, E., Burt, D., Gagliardi, C., Cioffi, G., Martelli, S., Perrett, D., et al. (2006). Is everybody always my friend? Perception of approachability in Williams syndrome. Neuropsychologia, 44, 254-259.

Gagliardi, C., Frigerio, E., Burt, D., Cazzaniga, I., Perrett, D., \& Borgatti, R. (2003). Facial expression recognition in Williams syndrome. Neuropsychologia, 41(6), 733738.

Gagliardi, C., Martelli, S., Burt, M., \& Borgatti, R. (2007). Evolution of neurologic features in Williams syndrome. Pediatric Neurology, 36, 301-306.

Galaburda, A., \& Bellugi, U. (2000). V. Multi-level analysis of cortical neuroanatomy in Williams syndrome. Journal of Cognitive Neuroscience, 12(Suppl. 1), 74-88.

Gaser, C., Luders, E., Thompson, P., Lee, A., Dutton, R., Geaga, J., et al. (2006). Increased local gyrification mapped in Williams syndrome. Neuroimage, 33, 46-54.

Gothelf, D., Searcy, Y., Reilly, J., Lai, P., Lanre-Amos, T., Mills, D., et al. (2008). Association between cerebral shape and social use of language in Williams syndrome. American Journal of Medical Genetics Part A, 146, 2753-2761.

Haas, B., Hoeft, F., Searcy, Y., Mills, D., Bellugi, U., \& Reiss, A. (2009). Individual differences in social behavior predict amygdala response to fearful facial expressions in Williams syndrome. Neuropsychologia

Jernigan, T., Bellugi, U., Sowell, E., Doherty, S., \& Hesselink, J. (1993). Cerebral morphologic distinctions between Williams and Down syndromes. Archives of Neurology - Chicago, 50, 186-1186.

Jones, W., Bellugi, U., Lai, Z., Chiles, M., Reilly, J., Lincoln, A., et al. (2000). II. Hypersociability in Williams syndrome. Journal of Cognitive Neuroscience, 12(Suppl. 1), 30-46.

Kaufman, A., Kaufman, J., Balgopal, R., \& McLean, J. (1996). Comparison of three WISC-III short forms: Weighing psychometric, clinical, and practical factors. Journal of Clinical Child E' Adolescent Psychology, 25(1), 97-105.

Klein-Tasman, B., \& Mervis, C. (2003). Distinctive personality characteristics of 8-, 9-, and 10-year-olds with Williams syndrome. Developmental Neuropsychology, 23(1), 269-290.

Lacroix, A., Guidetti, M., Rogé, B., \& Reilly, J. (2009). Recognition of emotional and nonemotional facial expressions: A comparison between Williams syndrome and autism. Research in Developmental Disabilities, 30, 976-985.

Liddle, P., Kiehl, K., \& Smith, A. (2001). Event-related fMRI study of response inhibition. Human Brain Mapping, 12(2), 100-109.

Lundqvist, D., Flykt, A., \& Öhman, A. (1998). The Karolinska directed emotional faces.

Martens, M., Wilson, S., Dudgeon, P., \& Reutens, D. (2009). Approachability and the amygdala: Insights from Williams syndrome. Neuropsychologia, 47(12), 24462453.

Menghini, D., Addona, F., Costanzo, F., \& Vicari, S. (2010). Executive functions in individuals with Williams syndrome. Journal of Intellectual Disability Research, 54(5), 418-432.

Meyer-Lindenberg, A., Hariri, A., Munoz, K., Mervis, C., Mattay, V., Morris, C., et al. (2005). Neural correlates of genetically abnormal social cognition in Williams syndrome. Nature Neuroscience, 8(8), 991-993.

Meyer-Lindenberg, A., Kohn, P., Mervis, C., Kippenhan, J., Olsen, R., Morris, C., et al. (2004). Neural basis of genetically determined visuospatial construction deficit in Williams syndrome. Neuron, 43(5), 623-631.

Mobbs, D., Eckert, M. A., Mills, D., Korenberg, J., Bellugi, U., Galaburda, A. M., et al. (2007). Frontostriatal Dysfunction During Response Inhibition in Williams Syndrome. Biological Psychiatry, 62, 256-261.

Muñoz, K., Meyer-Lindenberg, A., Hariri, A., Mervis, C., Mattay, V., Morris, C., et al. (2009). Abnormalities in neural processing of emotional stimuli in Williams syndrome vary according to social vs. non-social content. Neuroimage .

Paul, B., Snyder, A., Haist, F., Raichle, M., Bellugi, U., \& Stiles, J. (2009). Amygdala response to faces parallels social behavior in Williams syndrome. Social Cognitive and Affective Neuroscience.

Peoples, R., Franke, Y., Wang, Y. K., Perez-Jurado, L., Paperna, T., Cisco, M., et al. (2000). A physical map, including a BAC/PAC clone contig, of the Williams-Beuren syndrome - deletion region at 7q11.23. American Journal of Human Genetics, 66(1), 47-68.

Porter, M., Coltheart, M., \& Langdon, R. (2007). The neuropsychological basis of hypersociability in Williams and Down syndrome. Neuropsychologia, 45(12), 28392849.

Powell, T., \& Malia, K. (2003). The Brain Injury Workbook: Exercises for Cognitive Rehabilitation. London: Speechmark.

Reiss, A., Eckert, M., Rose, F., Karchemskiy, A., Kesler, S., Chang, M., et al. (2004). An experiment of nature: Brain anatomy parallels cognition and behavior in Williams syndrome. Journal of Neuroscience, 24(21), 5009.

Reynolds, D., \& Jeeves, M. (1978). A developmental study of hemisphere specialization for recognition of faces in normal subjects. Cortex; A Journal Devoted to the Study of the Nervous System and Behavior, 14(4), 511

Rhodes, S., Riby, D., Park, J., Fraser, E., \& Campbell, L. (2009). Executive neuropsychological functioning in individuals with Williams syndrome. Neuropsychologia .

Schmitt, J., Watts, K., Eliez, S., Bellugi, U., Galaburda, A., \& Reiss, A. (2002). Increased gyrification in Williams syndrome: Evidence using 3D MRI methods. Developmental Medicine E' Child Neurology, 44(5), 292-295.

Schoenbaum, G., Nugent, S., Saddoris, M., \& Setlow, B. (2002). Orbitofrontal lesions in rats impair reversal but not acquisition of go, no-go odor discriminations. Neuroreport, 13(6), 885.

Schulz, K., Fan, J., Magidina, O., Marks, D., Hahn, B., \& Halperin, J. (2007). Does the emotional go/no-go task really measure behavioral inhibition?: Convergence with measures on a non-emotional analog. Archives of Clinical Neuropsychology, 22(2), 151-160.

Semel, S. R., \& Rosner, E. (2003). Understanding Williams syndrome: Behavioral patterns and interventions mahwah. NJ: Lawrence Erlbaum Associates.

Skwerer, D., Borum, L., Verbalis, A., Schofield, C., Crawford, N., Ciciolla, L., et al. (2009). Autonomic responses to dynamic displays of facial expressions in adolescents and adults with Williams syndrome. Social Cognitive and Affective Neuroscience, 4(1), 93.

Tager-Flusberg, H., \& Sullivan, K. (2000). A componential view of theory of mind: Evidence from Williams syndrome. Cognition, 76(1), 59-90.

Terman, L. (1916). The uses of intelligence tests. The Measurement of Intelligence, 3-21.

Wechsler, D. (1991). Wechsler intelligence scale for children. Manual (3rd ed.). San Antonio: Psychological Corporation.

Wechsler, D. (1997). Wechsler adult intelligence scale-III (WAIS-III). San Antonio, TX: The Psychological Corporation.

Wechsler, D., \& Corporation, P. (2006). WPPSI-R, manual: Wechsler preschool and primary scale of intelligence - Revised. Dansk Psykologisk Forlag. 